July 23, 2018

LBNL-56805

NSF-KITP-05-02

\title{
Further Results About Field Theory On The World Sheet And String Formation ${ }^{1}$
}

\author{
Korkut Bardakci \\ Department of Physics \\ University of California at Berkeley \\ and \\ Theoretical Physics Group \\ Lawrence Berkeley National Laboratory \\ University of California \\ Berkeley, California 94720
}

\begin{abstract}
The present article is the continuation of the earlier work, which used the world sheet representation and the mean field approximation to sum planar graphs in massless $\phi^{3}$ field theory. We improve on the previous work in two respects: A prefactor in the world sheet propagator that had been neglected is now taken into account. In addition, we introduce a non-zero bare mass for the field $\phi$. Working with a theory with cutoff and using the mean field approximation, we find that, depending on the range of values of the mass and coupling constant, the model has two phases: A string forming phase and a perturbative field theory phase. We also find the generation of a new
\end{abstract}

\footnotetext{
${ }^{1}$ This work was supported in part by the Director, Office of Science, Office of High Energy and Nuclear Physics, of the U.S. Department of Energy under Contract DE-AC0376SF00098, and in part by the National Science Foundation Grant No.PHY99-07949
} 
degree of freedom, which was not in the model originally. This new degree of freedom can be thought of as the string slope, which is now promoted into a fluctuating dynamical variable. Finally, we show that the introduction of the bare mass makes it possible to renormalize the model. 


\section{Introduction}

The present article is the continuation of a series of articles $[1,2,3,4,5]$ pursuing a program of summing planar graphs in field theory. Because of its simplicity, the theory most intensively investigated so far is the $\phi^{3}$ theory, although progress has been made in extending the program to more physical theories $[6,7,8]$. The basic idea, due to 't Hooft [9], is to represent planar Feynman graphs on the world sheet, using a mixture of position and momentum space light cone variables as coordinates. This representation, which was originally non-local, was later reformulated as a local field theory on the world sheet by introducing additional non-dynamical fields [1]. The advantage of this reformulation is that it provides a useful setup for studying the sum of planar Feynman graphs, since one can then appeal to various approximation schemes familiar from field theory to investigate problems of interest.

The problem we are going to investigate in this article is string formation in the $\phi^{3}$ field theory and the approximation scheme we are going to use is the mean field method. Although the mean field method has its limitations, we hope that at least the predictions it makes about basic dynamical questions such as string formation are qualitatively correct. In any case, it is a simple method to use and we have nothing better available at the present.

The present paper can be viewed as a follow up to reference [5]. As such, it has a good deal of overlap with it, as well as with some of the earlier work on the same subject. This is because in organizing this paper, the goal was to present a self contained treatment, which should be intelligible even to a reader unfamiliar with the previous work on the subject. When we preview the rest of the paper below, we will try to make clear what is new and what is a review of the earlier work cited at the beginning of this section.

Before getting started, it may be helpful to summarize the advances made in this paper in comparison to [5]. Apart from some simplification and streamlining of the treatment, there are two new features of interest. The first one is somewhat technical: The prefactor $1 /\left(2 p^{+}\right)$in eq. $(2)$ for the propagator was neglected in the previous applications of the mean field method. In the present work, this factor is taken into account. This does effect the details of calculations, but it does not qualitatively change the final results, concerning, for example, string formation.

The second new feature is more significant. In reference [5], as well as in the work preceding it, the field theoretic bare mass was taken to be zero. 
Of course, in any case, a non-zero mass is generated in higher orders of perturbation theory, which is cutoff dependent and needs renormalization. This cutoff dependence shows up in the expression for the ground state energy (eq.(56)), which is proportional to the cutoff parameter $1 / a^{2}$. In the present work, we show how to introduce a non-zero bare mass, which can then be used as a counter term to cancel the cutoff dependent part of the ground state energy.

We would like to draw the attention of the reader to the main results obtained in this article. We show that, in the cutoff theory prior to renormalization, there is string formation for large enough values of the coupling constant, whereas for smaller values of the coupling constant, the model is in the perturbative field theory phase. We also show that physical quantities can be made cutoff independent by introducing a suitable bare mass. From the string perspective, the physical quantities we are referring to are the string slope and the intercept. With the introduction of mass, there is again string formation for a range of values of mass and coupling constant, and field theory phase takes over when the parameters are outside this range. An important problem not addressed in this paper is whether string formation observed in the cutoff theory persists after renormalization.

In section 2, we briefly review both the rules for Feynman graphs in the light cone variables [9] and the local field theory on the world sheet which generates these graphs [1]. We also discuss the transformation properties of the fields under a special Lorentz boost, which manifests itself as a scale transformation on the world sheet. Since lack of invariance under this scaling would imply violation of Lorentz invariance, as we go along, we make sure that no such violation occurs. This is an abridged version of a more complete discussion given in [4].

In the local field theory discussed in section 2 , the boundary conditions on the fields were imposed by hand. In section 3, a more general field theory is constructed, where the boundary conditions are enforced by means of Lagrange multipliers. In addition, auxilliary fermionic fields that distinguish between the boundaries and the bulk are needed. To have a well defined theory, we have to introduce two distinct cutoffs; one associated with the coordinate $\tau$ and the other with $\sigma$. Until section 8, we will be working exclusively with the cutoff theory, and all the results obtained will refer to this theory. The motivation for studying the cutoff theory is that it is an interesting model in its own right and also it is an indispensible preliminary to renormalization. This section follows reference [5] closely, with 
the exception that, unlike in [5], we do not impose supersymmetry on the model. Although the use supersymmetry is an elegant way of taking care of the matter-ghost cancellations, in retrospect, ghosts do not contribute in any significant way to the meanfield calculations. In the interests brevity and simplicity, we have therefore decided to drop the ghost sector and write down a non-supersymmetric model.

The mean field method which is at the basis of the present work is discussed in section 4 from the point of view of the large $D$ limit, where $D$ is the dimension of the transverse space. This section is largely a review of the material developed in the earlier work. The only thing new is a brief comparison of the determinant resulting from the integration over matter fields with the corresponding result well known from string theory.

In section 5, the fermionic part of the action is diagonalized, and the two fermionic energy levels are calculated as a function of a parameter $x$, which serves as an order parameter. It is argued that, independent of any approximation scheme, a non-zero order parameter signals both string formation and condensation of Feynman graphs on the world sheet, thereby linking these two phenomena. On the other hand, $x=0$ corresponds to the perturbative field theory phase of the model. This section is a mostly a review of the material covered in the previous work.

In section 6 , the prefactor $1 /\left(2 p^{+}\right)$in the propagator (eq.(2)), neglected in the previous work, is taken into account in the leading order of the mean field approximation. As a result, the interaction vertex becomes a function of the order parameter $x$ (eq.(52)). The material covered in this section is completely new.

In section 7 , combining the results of the previous sections, the ground state energy is expressed in terms of $x$. Minimizing this energy, we find that there is a critical value of the coupling constant, $g=g_{c}$ : For $g<g_{c}$, the minimum is at $x=0$, whereas for $g>g_{c}$, the minimum is at some $x \neq 0$. It then follows that, at $g=g_{c}$, there is a transition from the perturbative field theory phase to the string phase. A considerable portion of this section is new.

So far, we have been studying a model where the field theory bare mass was set equal to zero. In section 8, we show how to introduce a non-zero bare mass term in the leading order of the mean field approximation, and we show that, if the mass is not too large, the same picture as in the massless case emerges: There is a $g_{c}$, dependent on the mass, that seperates the string forming and perturbative phases of the model. It is also possible to use 
the bare mass as a counter term to eliminate the cutoff dependence of the ground state energy. From the string perspective, this corresponds to the renormalization of the intercept. Since, in section 5, we have already shown that the string slope is already finite, at least for a free string, this is all the renormalization that is needed. The investigation of the properties of the renormalized theory, which could be quite different from those of the cutoff theory studied so far, is left for future research. This section is completely new.

All the results obtained so far were in the leading order of the mean field approximation, or what is the same thing, in the leading order of large $D$ limit. In section 9, we compute a particular contribution coming from the next order of the large $D$ limit. This contribution is important in that it provides the kinetic energy term for a new propagating degree of freedom which was not present in the original action. As a result, the string slope, which was a constant in the leading order, becomes a dynamical field which can fluctuate around its mean value. We end the section with some speculative remarks about the possible connection between this new dynamical field and the extra dimension in AdS/CFT correspondence [10,11], and how it could possibly interpolate between "hard" and "soft" high energy processes. This section largely overlaps with the corresponding material in [5]; it is included here for the sake of completeness.

Finally, Appendices A and B contain some of the additional details of the mean field calculations developed in sections 6 and 8 .

\section{A Brief Review}

The Feynman graphs of a massive $\phi^{3}$ theory have a particularly simple form in the mixed lightcone representation of 't Hooft [9]. In this representation, the evolution parameter, $\tau$, is $x^{+}$, and the conjugate Hamiltonian is $p^{-}$. The Minkowski evolution operator is given by

$$
T=\exp \left(-i x^{+} p^{-}\right)
$$

The notation is such that a Minkowski four vector $v^{\mu}$ has the light cone components $\left(v^{+}, v^{-}, \mathbf{v}\right)$, where $v^{ \pm}=\left(v^{0} \pm v^{1}\right) / \sqrt{2}$, and boldface letters label the transverse directions. A propagator that carries momentum $p$ is pictured as a horizontal strip of width $p^{+}$and length $\tau=x^{+}$, bounded by two solid lines on the world sheet (Fig.1). The solid lines forming the boundary carry 


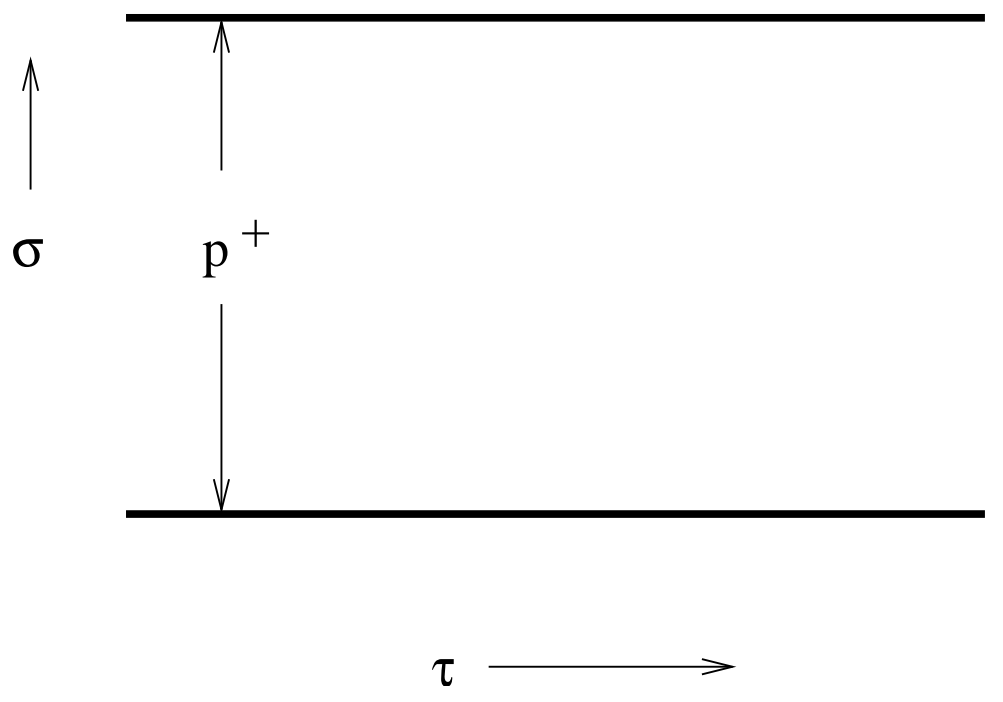

Figure 1: The Propagator

transverse momenta $\mathbf{q}_{1}$ and $\mathbf{q}_{2}$, with

$$
\mathbf{p}=\mathbf{q}_{1}-\mathbf{q}_{2},
$$

and the corresponding propagator is given by

$$
\Delta(p)=\frac{\theta(\tau)}{2 p^{+}} \exp \left(-i \frac{\tau}{2 p^{+}}\left(\mathbf{p}^{2}+m^{2}\right)\right) .
$$

More complicated graphs consist of several horizontal solid line segments (Fig.2). A factor of $g$, the coupling constant, is associated with the beginning and the end of each line segment, where the interaction takes place. Finally, one has to integrate over the positions of the interaction vertices, as well as the momenta carried by the solid lines. A typical light cone graph is pictured in Fig.2.

It was shown in [1] that the light cone Feynman rules described above can be reproduced by a local world sheet field theory. The world sheet is parametrized by the coordinates $\sigma$ along the $p^{+}$direction and $\tau$ along the $x^{+}$direction, and the transverse momentum $\mathbf{q}$ is promoted to a bosonic field $\mathbf{q}(\sigma, \tau)$ on the world sheet. In addition, two fermionic fields (ghosts) $b(\sigma, \tau)$ and $c(\sigma, \tau)$ are needed. In contrast to $\mathbf{q}$, which has $\mathrm{D}$ components, $b$ and $c$ each have $D / 2$ components. Here, $\mathrm{D}$ is the dimension of the transverse 


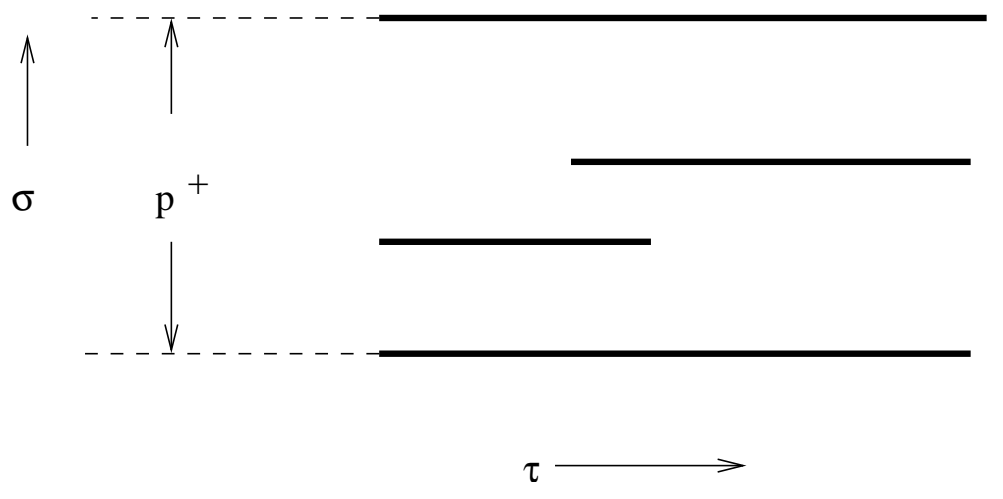

Figure 2: A Typical Graph

space, assumed to be even. Here and in the next section, we will consider first consider the zero mass case for the sake of simplicity, and postpone the discussion of the the massive theory to section (8). The action on the world sheet for the massless theory $(m=0)$, is given by

$$
S_{0}=\int_{0}^{p^{+}} d \sigma \int_{\tau_{i}}^{\tau_{f}} d \tau\left(b^{\prime} \cdot c^{\prime}-\frac{1}{2} \mathbf{q}^{\prime 2}\right),
$$

where the prime denotes derivative with respect to $\sigma$. This action is supplemented by Dirichlet boundary conditions

$$
\dot{\mathbf{q}}=0, b=c=0,
$$

on the solid lines, where the dot denotes derivative with respect to $\tau$. It was shown in [1] that if the equations of motion for $\mathbf{q}$ are solved subject to the above boundary conditions, the resulting classical action reproduces the exponential factor in eq.(2). The unwanted quantum contribution

$$
-\frac{D}{2} \operatorname{det}\left(\partial_{\sigma}^{2}\right)
$$

is cancelled by the corresponding determinant resulting from integrating over ghosts.

The action formulation described above was extensively used in the previous work $[2,3,4,5]$. It has, however, two defects: The prefactor $1 /\left(2 p^{+}\right)$in eq.(2) is missing and a non-zero bare mass cannot be accomodated. Since there is no symmetry forcing the renormalized mass to be zero, this means 
that the mass counter term needed for renormalization cannot be introduced. In sections (6) and (8), we will show how to overcome both of these problems in the context of the mean field approximation.

Finally, we would like to discuss briefly the question of Lorentz invariance. This is a non-trivial problem, since the use of the light cone variables obscures the Lorentz transformation properties of the fields. There is, however, a special subgroup of the Lorentz group, under which the light cone coordinates have simple linear transformation properties. If $L_{i, j}$ are the angular momenta and $K_{i}$ are the boosts, the generators of this subgroup are

$$
L_{i, j}, \quad M_{+,-}=K_{1}, \quad M_{+, i}=K_{i}+L_{1, i}
$$

where the indices $i$ and $j$ run from 2 to $D+2$. It turns out that invariance under all the generators, with the sole exception of $K_{1}$, is rather trivial [4]. The non-trivial transformation generated by $K_{1}$ corresponds to scaling of $x^{+}$ and $p^{+}$by a constant $\mathrm{u}$ :

$$
x^{+} \rightarrow x^{+} / u, \quad p^{+} \rightarrow p^{+} / u,
$$

leaving the transverse momenta $\mathbf{q}$ unchanged. As we go along, we will check the invariance the invariance of our equations and our results under this scale transformation. However, in this paper we will not investigate the problem of invariance under the full Lorentz group ${ }^{2}$

Let us check the scale invariance of (2) and (3). In eq. (2), the exponential is clearly invariant, and the prefactor $1 /\left(2 p^{+}\right)$is the integration measure that makes integration over $p^{+}$invariant. The action (3) and the boundary conditions (4) are also invariant if the fields transform according to

$$
\mathbf{q}(\sigma, \tau) \rightarrow \mathbf{q}(u \sigma, u \tau), \quad b, c(\sigma, \tau) \rightarrow b, c(u \sigma, u \tau)
$$

\section{The Complete World Sheet Action For $\mathbf{m}=0$}

It is possible to include the boundary conditions of eq.(4) in the action itself, rather then imposing them by hand $[2,3,4]$. What follows is a condensed version of the treatment given in $[3,4]$. The complete world sheet action in the zero mass case can be written as a sum of four terms:

$$
S=S_{m}+S_{g}+S_{g . f}+S_{f}
$$

\footnotetext{
${ }^{2}$ The problems of Lorentz invariance and renormalization are closely related. See [12] for an investigation of both problems in the light cone formalism.
} 
$S_{m}$, the matter action, and $S_{g}$, the ghost action, are given by

$$
\begin{aligned}
S_{m} & =\int_{0}^{p^{+}} d \sigma \int_{\tau_{i}}^{\tau_{f}} d \tau\left(-\frac{1}{2} \mathbf{q}^{2}+\rho \mathbf{y} \cdot \dot{\mathbf{q}}\right), \\
S_{g} & =\int_{0}^{p^{+}} d \sigma \int_{\tau_{i}}^{\tau_{f}} d \tau\left(b^{\prime} \cdot c^{\prime}+\rho \bar{b} \cdot b+\rho \bar{c} \cdot c\right) .
\end{aligned}
$$

Here the boundary conditions on the matter and ghost fields are implemented by means of the Lagrange multiplier fields $\mathbf{y}, \bar{b}$ and $\bar{c}$. The field $\rho$ is a delta function on the boundaries(solid lines) and zero in the bulk, in order to ensure that the boundary conditions are imposed on the boundaries and not in the bulk. We will shortly give an explicit construction for $\rho$ in terms of fermionic fields. $S_{g . f}$ is the gauge fixing term given by

$$
S_{g . f}=\int_{0}^{p^{+}} d \sigma \int_{\tau_{i}}^{\tau_{f}} d \tau\left(-\frac{1}{2} \bar{\rho} \alpha^{2} \mathbf{y}^{2}\right),
$$

where $\alpha$ is a constant and $\bar{\rho}$ is complementary to $\rho$; it vanishes on the boundaries and is equal to one everywhere else. In the absence of this term, the integration over over $\mathbf{y}$ the action is invariant under a gauge transformation of the form

$$
\mathbf{y} \rightarrow \mathbf{y}+\mathbf{z} \bar{\rho},
$$

where $\mathbf{z}$ is an arbirary function of the coordinates. This gauge invariance causes the functional integral over $\mathbf{y}$ to diverge in the bulk where $\bar{\rho}$ is zero; fixing the gauge eliminates this divergence. There is also a Faddeev-Popov type measure factor associated with gauge fixing. One can see the need for it as follows: The integral over $\mathbf{y}$ is Gaussian away from the boundaries and it can explicitly be evaluated, resulting in a singular contribution that depends on the gauge fixing parameter $\alpha$. If we regulate this singular expression by discretizing the $\tau$ coordinate into segments of length $a^{\prime}$, we have a product of the form

$$
\prod\left(a^{\prime} \alpha^{2}\right)^{-D / 2} .
$$

This is an unwanted factor resulting from gauge fixing. It can be cancelled by introducing a compensating measure factor in the $\mathbf{y}$, or by choosing

$$
a^{\prime} \alpha^{2}=1 .
$$

From now on, we will assume that $\alpha$ is fixed by this relation. We would like to point out that in the earlier work, for example reference [5], $\alpha$ was 


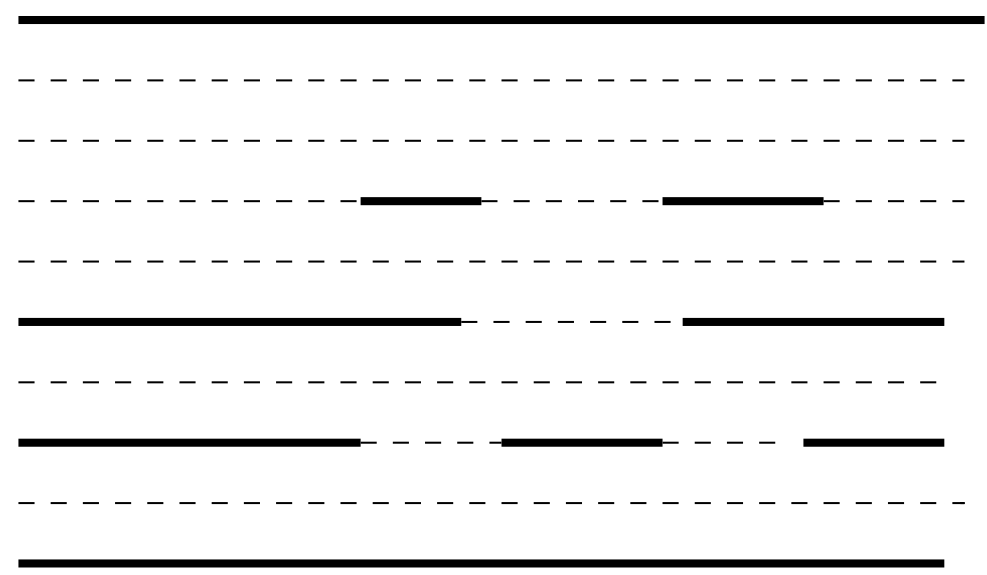

Figure 3: Solid and Dotted Lines

left arbitrary. It turns out that as a consequence of eq.(28), the string slope parameter, eq.(42) comes out finite without any need of renormalization.

It remains to specify the fermionic action $S_{f}$. Introducing a two component fermion field $\psi_{i}(\sigma, \tau), i=1,2$, and its adjoint $\bar{\psi}_{i}(\sigma, \tau), \rho$ and $\bar{\rho}$ of eqs. $(9,10)$ can be expressed as

$$
\rho=\frac{1}{2} \bar{\psi}\left(1-\sigma_{3}\right) \psi, \quad \bar{\rho}=\frac{1}{2} \bar{\psi}\left(1+\sigma_{3}\right) \psi
$$

To see how this works, it is best to discretize the $\sigma$ coordinate into segments of length $a$, which also helps regulate the fermionic sector. This discretization is pictorially represented in Fig.3 by horizontal lines at constant $\sigma$, spaced distance $a$ apart. The boundaries are marked by solid lines as before, and the rest of the space (the bulk) is filled with dotted lines. If we identify the spin up component of the fermionic field $(i=1)$ with the dotted lines and the spin down component $(i=2)$ with the solid lines, $\rho$ and $\bar{\rho}$ are then the spin down and spin up projection operators. Therefore, $\rho=1$ on solid lines and it is zero on dotted lines, as stated before, and the opposite holds for $\bar{\rho}$.

We have so far introduced two cutoff parameters, $a$ and $a^{\prime}$. All the results obtained until section 8 refer to the cutoff model regularized by these two parameters. In section 8, we will show how the cutoff dependence can be eliminated by means of mass renormalization. Unfortunately, in this paper, we will not have much to say about the renormalized model. 
With these preliminaries out of the way, the fermionic action is given by

$$
\begin{aligned}
S_{f} & =\sum_{n} \int d \tau\left(i \bar{\psi}_{n} \dot{\psi}_{n}-\tilde{g} D \bar{\psi}_{n} \sigma_{1} \psi_{n}\right) \\
& \rightarrow \int_{0}^{p^{+}} d \sigma \int_{\tau_{i}}^{\tau_{f}} d \tau\left(i \bar{\psi} \dot{\psi}-\tilde{g} D \bar{\psi} \sigma_{1} \psi\right)
\end{aligned}
$$

The first line is the action in terms of discretized fermions

$$
\psi_{n}(\tau)=\psi(n a, \tau),
$$

and the second line is in terms of continuum fermions. Since the canonical anticommutation relations are in terms of a Kroenecker delta in the first case and a Dirac delta in the second case, these two fermions differ in normalization by a factor of $\sqrt{a}$. The first term in the action represents the time propagation of the free fermion without spin flip, the solid and dotted lines propagating unchanged. The second term, in flipping the spin, converts a solid line into a dotted line and vice versa. This spin flip represents the $\phi^{3}$ interaction and it is accompanied by the coupling constant $\tilde{g}$, which we have scaled by a factor of $\mathrm{D}$ for later convenience. As it will become clear later, this is necessary to have a non-trivial large $D$ limit; otherwise, the theory would be non-interacting in this limit. Note that, with this scaling, large $D$ is the same as strong coupling. Now if $\tilde{g}$ is taken to be a constant, as was done in the earlier work $[3,4,5]$, then the world sheet field theory would reproduce only the exponential in eq. $(2)$, and the factor $1 /\left(2 p^{+}\right)$would be missing. Later, we will show how to take care of this factor in the mean field approximation by allowing it to become a function of the dynamical variables.

We will now rewrite the full action, collecting the terms for $S_{m}, S_{g . f}$ and $S_{f}$, but excluding $S_{g}$. We will argue later that the inclusion of $S_{g}$ does not affect the dynamics of the problem. With this omission, $S$ is given by

$$
\begin{aligned}
S & =\int_{0}^{p^{+}} d \sigma \int_{\tau_{i}}^{\tau_{f}} d \tau\left(-\frac{1}{2} \mathbf{q}^{\prime 2}+i \bar{\psi} \dot{\psi}-\tilde{g} D \bar{\psi} \sigma_{1} \psi+\frac{1}{2} \mathbf{y} \cdot \dot{\mathbf{q}} \bar{\psi}\left(1-\sigma_{3}\right) \psi\right. \\
& \left.-\frac{1}{4} \alpha^{2} \mathbf{y}^{2} \bar{\psi}\left(1+\sigma_{3}\right) \psi\right) .
\end{aligned}
$$

Let us check the scale invariance of this action. If under scaling, the fields $\mathbf{q}$, $\mathbf{y}$ and the fermions transform as

$$
\begin{aligned}
& \mathbf{q}(\sigma, \tau) \rightarrow \mathbf{q}(u \sigma, u \tau), \mathbf{y}(\sigma, \tau) \rightarrow \mathbf{y}(u \sigma, u \tau), \\
& \psi(\sigma, \tau) \rightarrow \sqrt{u} \psi(u \sigma, u \tau), \bar{\psi}(\sigma, \tau) \rightarrow \sqrt{u} \bar{\psi}(u \sigma, u \tau),
\end{aligned}
$$


then all the terms are invariant except the interaction term proportional to $\tilde{g}$ and the gauge fixing term proportional to $\alpha^{2}$. These two terms become invariant only by demanding that $\tilde{g}$ and $\alpha^{2}$ transform by

$$
\tilde{g} \rightarrow u \tilde{g}, \quad \alpha^{2} \rightarrow u \alpha^{2}
$$

We will eventually fix $\alpha^{2}$ by setting $a^{\prime} \alpha^{2}=1$, as in eq.(11). This brings up the question whether the lattice spacings $a$ and $a^{\prime}$ in the $\sigma$ and $\tau$ directions transform under scaling. It is unusual to assign transformation properties to a cutoff; however, we will argue that in this case it is quite natural. For example, if we split the interval from $\sigma=0$ to $\sigma=p^{+}$in $\mathrm{N}$ segments of length $a$, it is clear that under scaling, $\mathrm{N}$, being an integer, does not change, and therefore, $a$ must transform like $p^{+}$. A similar argument applies to $a^{\prime}$, so we must have

$$
a \rightarrow a / u, \quad a^{\prime} \rightarrow a^{\prime} / u
$$

under scaling. This shows that $\alpha^{2}$, fixed by eq.(11), scales correctly. We will see that the same is true for $\tilde{g}$ in section (6).

\section{The Meanfield Approximation}

The meanfield method as applied to this problem was developed in [3] and [5]. We will mainly follow the treatment given in [5], identifying the mean field method with the large $D$ limit. Unlike in [5] however, there will be no supersymmetry on the world sheet. We notice that the action (14) represents a vector model, which can be solved in the large $D$ limit [13]. The standard approach is to replace the scalar products of the vector fields $\mathbf{y}$ and $\mathbf{q}$, namely $\mathbf{y} \cdot \dot{\mathbf{q}}$ and $\mathbf{y}^{2}$, by their vacuum expectation values. The functional integral over the remaining fields is carried out exactly, and the resulting effective action is minimized with respect to the vacuum expectation values. An efficient way of carrying out this program is to introduce two composite fields $\lambda_{1}$ and $\lambda_{2}$ by adding a term $\Delta S$ to the action:

$$
\begin{aligned}
S & \rightarrow S+\Delta S \\
\Delta S & =\int_{0}^{p^{+}} d \sigma \int_{\tau_{i}}^{\tau_{f}} d \tau\left(\kappa_{1}\left(D \lambda_{1}-\mathbf{y} \cdot \dot{\mathbf{q}}\right)+\frac{\kappa_{2}}{2}\left(D \lambda_{2}-\mathbf{y}^{2}\right)\right),
\end{aligned}
$$

where $\kappa_{1,2}$ act as Lagrange multipliers. All we have done is to rename the composite fields $\mathbf{y} \cdot \dot{\mathbf{q}}$ and $\mathbf{y}^{2}$ as $D \lambda_{1}$ and $D \lambda_{2}$. The factors of $D$ are natural since each of these composite fields is a sum of $D$ terms. After this renaming, 
the Gaussian integration over $\mathbf{y}$ can be done, and the action can be rewritten in the following form:

$$
\begin{aligned}
S & +\Delta S \rightarrow S_{1}+S_{2}+S_{3}, \\
S_{1} & =\int_{0}^{p^{+}} d \sigma \int_{\tau_{i}}^{\tau_{f}} d \tau\left(-\frac{1}{2} \mathbf{q}^{\prime 2}+\frac{\kappa_{1}^{2}}{2 \kappa_{2}} \dot{\mathbf{q}}^{2}\right), \\
S_{2} & =D \int_{0}^{p^{+}} d \sigma \int_{\tau_{i}}^{\tau_{f}} d \tau\left(\kappa_{1} \lambda_{1}+\frac{1}{2} \kappa_{2} \lambda_{2}\right), \\
S_{3} & =\int_{0}^{p^{+}} d \sigma \int_{\tau_{i}}^{\tau_{f}} d \tau\left(i \bar{\psi} \dot{\psi}-D \tilde{g} \bar{\psi} \sigma_{1} \psi+\frac{D}{2} \bar{\psi}\left(\lambda_{1}\left(1-\sigma_{3}\right)-\frac{1}{2} \alpha^{2} \lambda_{2}\left(1+\sigma_{3}\right)\right) \psi\right) .
\end{aligned}
$$

In the large $D$ limit, $\kappa_{1,2}$ and $\lambda_{1,2}$ can be replaced by their vacuum expectation values:

$$
\kappa_{1} \rightarrow \kappa_{1,0}=\left\langle\kappa_{1}\right\rangle, \quad \kappa_{2} \rightarrow \kappa_{2,0}=\left\langle\kappa_{2}\right\rangle, \quad \lambda_{1} \rightarrow \lambda_{1,0}=\left\langle\lambda_{1}\right\rangle, \quad \lambda_{2} \rightarrow \lambda_{2,0}=\left\langle\lambda_{2}\right\rangle,
$$

and therefore these fields become classical in this limit. In addition, an important simplification is achieved by setting the total momentum $\mathbf{p}$ carried by the whole graph equal to zero:

$$
\mathbf{p}=\int_{0}^{p^{+}} d \sigma \mathbf{q}^{\prime}=0
$$

This configuration, which can always be reached by a suitable Lorentz transformation, allows us to impose the periodic boundary conditions

$$
\mathbf{q}(\sigma=0, \tau)=\mathbf{q}\left(\sigma=p^{+}, \tau\right)
$$

The advantage of choosing this configuration is that it is translationally invariant in both the $\sigma$ and the $\tau$ directions, and consequently the classical fields $\kappa_{1,2}$ and $\lambda_{1,2}$ can be set equal to constants independent of coordinates. It then follows that

$$
A^{2}=\kappa_{1}^{2} / \kappa_{2} \rightarrow \kappa_{1,0}^{2} / \kappa_{2,0}
$$

tends to a constant in the limit of large $D$. We note that $S_{1}$ in eq.(19) is the standard string action, with the slope $\alpha^{\prime}$ given by

$$
\alpha^{\prime 2}=\frac{A^{2}}{4}=\kappa_{1,0}^{2} / 4 \kappa_{2,0}
$$


In general, this a fluctuating dynamical field, so it is far from clear that $S_{1}$ represents a real string with a constant slope. In the large $D$ limit, however, since $A^{2}$ tends to a constant, so does the slope, and, if this constant is positive and different from zero, a real string has formed. We will later see that this constant is never negative; however, it could vanish. In that case, we have a zero slope string theory, which is another name for a field theory. To conclude, there is string formation only if the ground state expectation value of $A^{2}$ is non-zero; otherwise, we have a field theory. Therefore, the ground state expectation value of $A^{2}$ serves as an order parameter that distinguishes between the field theory limit and string formation. In the leading large $D$ limit, this expectation value will turn out to be non-zero if the parameters of the model are in a suitable range, endowing the string with a constant nonzero slope. After the corrections to the large $D$ limit are taken into account, the string slope becomes dynamical and it can fluctuate.

If we replace $A^{2}$ by its constant expectation value, the functional integration over $\mathbf{q}$ in $S_{1}$ can easily be done, with the result

$$
\begin{aligned}
S_{1} & \rightarrow \frac{i}{2} D \operatorname{Tr} \ln \left(-\partial_{\sigma}^{2}+A^{2} \partial_{\tau}^{2}\right) \\
& =-\frac{D}{4 \pi}\left(\tau_{f}-\tau_{i}\right) \int d k \sum_{n \in Z} \ln \left(\left(\frac{2 \pi n}{p^{+}}\right)^{2}+A^{2} k^{2}\right) .
\end{aligned}
$$

This needs a ultraviolet cutoff in the variable $k$ to make sense, so we introduce a smooth cutoff function $f(k / \Lambda)$ by letting

$$
\int d k \sum_{n} \ln \left(\left(\frac{2 \pi n}{p^{+}}\right)^{2}+A^{2} k^{2}\right) \rightarrow \int d k f(k / \Lambda) \sum_{n} \ln \left(\left(\frac{2 \pi n}{p^{+}}\right)^{2}+A^{2} k^{2}\right) .
$$

This expression is not yet convergent, but the divergence is an additive constant independent of $A^{2}$. Since the meanfield equations only invove the derivative $S_{1}$ with respect to $A^{2}$, we can safely make the subtraction

$$
S_{1}\left(A^{2}\right) \rightarrow S_{1}\left(A^{2}\right)-S_{1}(0)
$$

and arrive at the finite result

$$
S_{1}\left(A^{2}\right)-S_{1}(0)=-\frac{D}{4 \pi}\left(\tau_{f}-\tau_{i}\right)\left(p^{+}|A| \int d k k f(k / \Lambda)-\frac{2 \pi^{2}}{3|A| p^{+}}\right) .
$$

In any case, if we did not drop the ghost action $S_{g}$, this additive constant would be cancelled by the contribution from the ghost sector [5]. 
The integral in the first term on the right is quadratic in the cutoff; for the sake of simplicity, we could just as well impose a sharp cutoff and set

$$
\int d k k f(k / \Lambda)=\Lambda^{2}
$$

In any case, a redefinition of the cutoff would yield the same result. In the rest of the paper, we will focus only on the cutoff dependent terms, and so, from now on, we will set

$$
S_{1} \simeq-\frac{D}{2 \pi}\left(\tau_{f}-\tau_{i}\right)|A| p^{+} \Lambda^{2}
$$

What we are doing is to study the cutoff theory prior to renormalization. The reason for doing so is twofold: The cutoff theory is of interest by itself; for example, in section 7, we will find string formation for a range of the values of the coupling constant. Also, we want to renormalize the ground state energy by introducing a mass counter term. To do this, we have to learn about the cutoff dependence of various quantities by first studying the cutoff theory.

Eq.(25) could also be obtained by appealing to standard results from string theory $[8,9]$. Scaling $\mathbf{q}$ by

$$
\mathbf{q} \rightarrow \mathbf{q} / A
$$

the $\operatorname{tr} \ln$ of eq. (23) is transformed into

$$
\operatorname{Tr} \ln \left(-\frac{1}{A} \partial_{\sigma}^{2}+A \partial_{\tau}^{2}\right)
$$

But the calculation of this $\operatorname{Tr} \ln$ is the same as calculating the ground state energy of a string with the constant background world sheet metric given by

$$
g^{0,0}=A, \quad g^{1,1}=1 / A, \quad g^{0,1}=g^{1,0}=0,
$$

and the result is the same as in eq.(25). In string theory, the cutoff dependent term, which contributes to the energy per unit length, is cancelled by a counter term. The finite term is the famous Casimir term which fixes the intercept.

Although there is this simple connection between our model and the standard string theory, we would like to emphasize that there are also significant 
differences. For example, the cutoff dependent term in string theory is a pure constant and it can be dropped without disturbing the dynamics. In contrast, the cutoff dependent term here is proportional to $A^{2}=\kappa_{1,0}^{2} / \kappa_{2,0}$, which is a dynamical quantity. Also, in our case, the coordinates $\sigma$ and $\tau$ are fixed once for all, and unlike in string theory, there is no general reparametrization invariance. For example, one cannot eliminate the dependence on $A$ by mapping the metric given by eq.(27) into

$$
g^{0,0}=g^{1,1}=1, \quad g^{0,1}=g^{1,0}=0 .
$$

We have so far introduced two different cutoffs in the $\tau$ direction; namely, $a^{\prime}$ in eq.(11) and $\Lambda$ in eq.(24). These are in fact related: If, for example, the momentum space conjugate to $\tau$ is compactified, the corresponding period can be identified with the cutoff $\Lambda$. The lattice spacing $a^{\prime}$ is then related to it by

$$
a^{\prime}=\frac{2 \pi}{\Lambda}
$$

\section{The Fermionic Action}

In this section, we will carry out the functional integral over the fermions in $S_{3}$, eq.(19), with $\lambda_{1}$ and $\lambda_{2}$ replaced by their coordinate independent expectation values, or the mean values, $\lambda_{1,0}$ and $\lambda_{2,0}$. To avoid divergences, we first regulate it by discretizing the $\sigma$ coordinate on a lattice of spacing $a$. There is then a complete decoupling of the different lattice sites, and at each

site, we have a two level quantum mechanics problem. Instead of working with the action, it is easier to diagonalize the corresponding Hamiltonian. The total Hamiltonian can be written as a sum of $N$ mutually commuting Hamiltonians, with $N=p^{+} / a$ :

$$
\begin{aligned}
H & =\sum_{n} H_{n} \\
H_{n} & =D\left(\tilde{g} \bar{\psi} \sigma_{1} \psi-\frac{1}{2} \bar{\psi}\left(\lambda_{1,0}\left(1-\sigma_{3}\right)-\frac{1}{2} \alpha^{2} \lambda_{2,0}\left(1+\sigma_{3}\right)\right) \psi\right)_{\sigma=\sigma_{n}} .
\end{aligned}
$$

Acting on spin up and spin down states (dotted and solid lines), $H_{n}$, the Hamiltonian at the site $\sigma=\sigma_{n}=n a$, reduces to a two by two matrix:

$$
H_{n} \rightarrow D\left(\begin{array}{cc}
\frac{1}{2} \alpha^{2} \lambda_{2,0} & \tilde{g} \\
\tilde{g} & -\lambda_{1,0}
\end{array}\right)
$$


Diagonalizing, we have the energy levels

$$
E_{n}^{ \pm}=\frac{D}{2}\left(\frac{1}{2} \alpha^{2} \lambda_{2,0}-\lambda_{1,0} \pm \sqrt{\left(\frac{1}{2} \alpha^{2} \lambda_{2,0}+\lambda_{1,0}\right)^{2}+4 \tilde{g}^{2}}\right) .
$$

In general, we expect the level corresponding to the minus sign to be energetically favored; however, we will keep both options open for the time being.

Eq.(31) gives the energy of a fermion located at a single lattice site $\sigma_{n}=$ $n a$; the total fermionic energy is gotten by multiplying this by $N=p^{+} / a$. Combining the fermionic contribution with those coming from $S_{1}$ and $S_{2}$, eqs.(26) and (19), the total action is given by

$$
\begin{aligned}
S^{ \pm} & =D p^{+}\left(\tau_{f}-\tau_{i}\right)\left(-\frac{2 \pi}{a^{\prime 2}}\left|\kappa_{1,0}\right| / \sqrt{\kappa_{2,0}}+\kappa_{1,0} \lambda_{1,0}+\frac{1}{2} \kappa_{2,0} \lambda_{2,0}\right. \\
& \left.-\frac{1}{2 a} \lambda_{-} \mp \frac{1}{2 a} \sqrt{\lambda_{+}^{2}+4 \tilde{g}^{2}}\right),
\end{aligned}
$$

where we have rewritten $A$ in terms of $\kappa$ 's (eq.(21)) and defined

$$
\lambda_{ \pm}=\frac{1}{2} \alpha^{2} \lambda_{2,0} \pm \lambda_{1,0} .
$$

Since this action is proportional to $D$, in the limit of large $D$, it can be evaluated using the saddle point method. This amounts to using the equations of motion in the action. Varying $\lambda_{-}$gives

$$
\kappa_{2,0}=\alpha^{2}\left(\kappa_{1,0}+\frac{1}{a}\right) .
$$

It is convenient to define the variable $\mathrm{x}$ by

$$
\kappa_{1,0}=-x / a,
$$

so that

$$
\kappa_{2,0}=\frac{\alpha^{2}}{a}(1-x) .
$$

$x$ will turn out to be positive, so we can drop the absolute value signs from now on. The equation of motion with respect to $\lambda_{+}$gives

$$
\lambda_{+}^{ \pm}= \pm \tilde{g} \frac{1-2 x}{\sqrt{x-x^{2}}},
$$


and the \pm signs in this equation are correlated with the \pm signs in eq.(31). Here, as well as in eq.(31), we have fixed the sign of $\tilde{g}$ to be positive, which can always be achieved by a redefinition of the \pm signs in front of it. Substituting these results into eq.(32), the corresponding energy, related to the action (32) by

$$
S^{ \pm}=-\left(\tau_{f}-\tau_{i}\right) E^{ \pm}
$$

can be written as

$$
E^{ \pm}=D p^{+}\left(\frac{2 \pi x^{ \pm}}{\alpha a^{\prime 2} \sqrt{a\left(1-x^{ \pm}\right)}} \pm \frac{2 \tilde{g}}{a} \sqrt{x^{ \pm}-\left(x^{ \pm}\right)^{2}}\right)
$$

In the first term on the right, the cutoff $\Lambda$ has been traded for $a^{\prime}$ through $\Lambda=2 \pi / a^{\prime}$ (eq.(28)). Here, $x^{ \pm}$are the values of $x$ that minimize the above total energy for the \pm solutions.

The eigenvectors of the Hamiltonian $H_{n}$ (eq.(29)) are also of interest. Denoting the normalized eigenvectors corresponding to \pm signs of the energy by

$$
\left(\begin{array}{c}
b_{1}^{ \pm} \\
b_{2}^{ \pm}
\end{array}\right)
$$

we have

$$
\begin{aligned}
& b_{1}^{ \pm}=\mp \sqrt{\frac{1}{2}\left(1 \pm \frac{\lambda_{+}}{\sqrt{\lambda_{+}^{2}+4 \tilde{g}^{2}}}\right)}=\mp \sqrt{1-x^{ \pm}}, \\
& b_{2}^{ \pm}=\sqrt{\frac{1}{2}\left(1 \mp \frac{\lambda_{+}}{\sqrt{\lambda_{+}^{2}+4 \tilde{g}^{2}}}\right)}=\sqrt{x^{ \pm}}
\end{aligned}
$$

Let us recall the physical significance of these matrix elements: The probabilities of having a dotted line (spin up) for the \pm solutions are given by

$$
\left(b_{1}^{ \pm}\right)^{2}=1-x^{ \pm}
$$

respectively. Similarly, the probabilities of having a solid line (spin down) for the \pm solutions are given by

$$
\left(b_{2}^{ \pm}\right)^{2}=x^{ \pm}
$$

From this probability interpretation for $x$, it follows that

$$
0 \leq x^{ \pm} \leq 1
$$


which we have already tacitly assumed. Otherwise, for example, eqs. $(35,36)$ would not make sense.

From eqs.(37), it is easy to show that

$$
\frac{1}{2}\left\langle\bar{\psi}\left(1-\sigma_{3}\right) \psi\right\rangle=\langle\rho\rangle=\frac{x^{ \pm}}{a}
$$

for both \pm solutions. We shall see below that (eq.(42)) $x$ is the order parameter that distinguishes between field theory and string theory: A non vanishing $x$ signals string formation, whereas $x=0$ corresponds to a zero slope string, which is another name for field theory. The equation above correlates $x$ with the expectation value of the fermionic bilinear $\bar{\psi}_{2} \psi_{2}$, so one could as well think of this bilinear as the order parameter. On the other hand this bilinear is the number operator that counts solid lines: A non vanishing expectation value for it means that a finite proportion of the area of the world sheet is covered by the solid lines; in other words, solid lines have condensed on the world sheet, leading to string formation. We would like to stress that this connection between condensation of solid lines on the world sheet and string formation is quite robust; it is valid independent of the approximation scheme used to compute $x^{-}$.

In the language of Feynman graphs, condensation of solid lines means that a single graph of asymptotically infinite order is dominating the sum over planar graphs. It is interesting to note that this was exactly the picture proposed in the very first papers that attempted to deduce string formation from Feynman graphs [14,15].

The next step is to determine $x^{ \pm}$by minimizing the total energy for each solution. Since both terms on the right hand side of eq.(36) are positive for the + solution and they have opposite signs for the - solution, we expect that - solution represents the ground state. We cannot quite calculate $x^{-}$ yet, since $\tilde{g}$ will turn out to depend on $x$, and we have first to determine this functional dependence. This will be done in the next section, but since, from eq. (34)

$$
\alpha^{\prime 2}=\frac{\kappa_{1,0}^{2}}{4 \kappa_{2,0}}=\frac{\left(x^{-}\right)^{2}}{4 a \alpha^{2}\left(1-x^{-}\right)},
$$

and taking $\alpha^{2}=1 / a^{\prime}$ (eq.(28)), then

$$
\alpha^{\prime 2}=\frac{a^{\prime}\left(x^{-}\right)^{2}}{4 a\left(1-x^{-}\right)} .
$$


Therefore, if $x^{-}$is non-zero, we can easily see that the slope parameter will also be non-zero ; conversely, $x^{-}=0$ means that the slope is zero. In reaching this conclusion, we have assumed, as we have done throughout this paper, that the ratio of the two lattice spacings, $a / a^{\prime}$, which is scale invariant (eq.(17)), is finite. Since this relation between the two cutoff parameters is essential for having a finite slope, we would like to argue that it is required by Lorentz invariance. In fact, if we instead allowed a more general relation, say,

$$
a^{\prime}=f(a),
$$

it is easy to see that, unless $f$ is of the form

$$
f(a)=c a
$$

where $c$ is a constant, invariance under scaling (eq.(17)) would be violated. This would in turn imply violation of Lorentz invariance. As a bonus, we end up with a finite slope, with no need of renormalization. In contrast, we shall see that the other parameter of string theory, the intercept, is cutoff dependent and needs renormalization. We note that there is nothing in the problem so far that fixes the ratio $a^{\prime} / a$, and therefore, a new parameter, in addition to the coupling constant, has to be introduced into the model. It is possible that the imposition of full Lorentz invariance will eventually fix this parameter.

What about the + solution? In this case, both terms are positive semidefinite, so clearly, $x^{+}=0$ minimizes the energy, and the probability of having a solid line is zero. This is the (trivial) starting point of standard perturbation theory; namely, no Feynman graphs and energy equal to zero. When higher order terms in $1 / D$ are taken into account, we expect $x^{+}$to fluctuate and allow the formation of solid lines, thereby generating higher order Feynman graphs. To summarize, $x$ could be non-zero only for the - solution, leading to string formation. On the other hand, the + solution always has $x=0$, corresponding to perturbative field theory. The ground state energy of the + solution is either greater than or equal to that of the - solution.

\section{The Interaction Vertex}

As we have stressed earlier, following eq.(13), taking $\tilde{g}$ to be a constant amounts to neglecting the factor of $1 /\left(2 p^{+}\right)$in the world sheet propagator (eq.(2)). We will now show that this factor can be taken into account in the leading mean field approximation, and as a result, $\tilde{g}$ becomes a function of the 


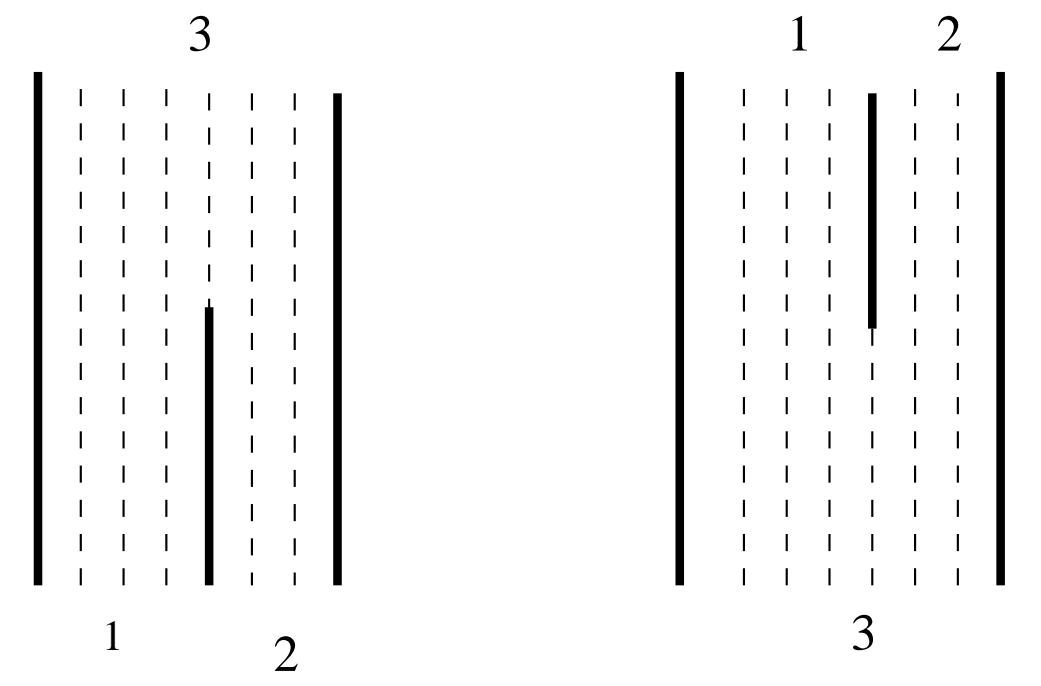

Figure 4: Two Vertices

variable $x$. We now proceed to calculate this function. It turns out that, for our purposes, it is more convenient to associate this factor $1 /\left(2 p^{+}\right)$with the vertices, rather than with the propagators. Consider two interaction vertices, with the propagators labeled 1,2 and 3 meeting at the vertex as shown in Fig.4. In one of them, a solid line turns into a dotted line, and in the other, the reverse takes place. With the first vertex, we associate a factor

$$
V_{+}=\frac{g}{8 p_{1}^{+} p_{2}^{+} p_{3}^{+}},
$$

and with the second vertex, a factor of

$$
V_{-}=g .
$$

Here, $g$ is related to the coupling constant of the $\phi^{3}$ interaction. It is easy to check that this is equivalent to assigning a factor of $1 /\left(2 p_{i}^{+}\right), i=1,2,3$, to each of the propagators labeled by $i$. This assignment is not symmetrical between $V_{+}$and $V_{-}$; but this is not a problem since only the product

$$
V=V_{+} V_{-}
$$

matters. For example, we could interchange the roles of these two vertices, or we could make a symmetrical assignment at the cost of introducing square 
roots. For the time being, the above assignment will be convenient to work with; later, we will show how to restore the symmetry between $V_{+}$and $V_{-}$.

At this point, one may wonder about the precise relationship between $g$ and the coupling constant of the $\phi^{3}$ interaction. Of course, this depends on renormalization, and therefore, to relate the coupling constant of field theory to that of the world sheet, one has to compare the renormalization schemes used in each case. Here we will simply treat $g$ as an effective coupling constant, and we will not try to compare it to the field theoretic constant. It is of interest to note that $g$ is a scale invariant constant, as contrasted to $\tilde{g}$ (eq.(16)), so it passes at least one important test for being a Lorentz scalar. It is also finite (cutoff independent), at least in the lowest order approximation. This is because, for example, if it depended on $a$ in non-trivially, it could not be scale invariant, since $a$ transforms under scaling (eq.(17)). So it may be more appropriate to think of $g$ as a renormalized coupling constant, rather than a bare one. Therefore, we have to do mass renormalization (section 8), but we do not have to worry about coupling constant renormalization.

Eq.(43) refers to a vertex where each leg carries a fixed momentum $p_{i}^{+}$. This means that when we write down the vertex in the language of field theory, we have to somehow express the $p^{+}$'s in terms of the local fields. To do this exactly is a difficult problem; however, there is a simple answer in the leading order of the meanfield approximation. In this approximation, we can replace the right hand side by its average value:

$$
V_{+} \rightarrow g\left\langle\frac{1}{8 p_{1}^{+} p_{2}^{+} p_{3}^{+}}\right\rangle \text {. }
$$

To compute the indicated average, one has to figure out the probability of occurence of a configuration with specified $p^{+}$'s. We recall from the last section that, in the leading order of the mean field approximation, the probability of having a dotted line is given by $1-x$ and that of having a solid line by $x$ (eqs. $(38,39))$. Here $x$ is a constant independent of the coordinates, to be determined by minimizing the ground state energy. Consider the configuration in Fig. 5 of the vertex $V_{+}$, where the momenta $p_{i}^{+}$discretized in steps of length $a$ as usual, with $n_{i}$ dotted lines associated with the propagator labeled by $i$, and

$$
p_{1}^{+}=\left(n_{i}+1\right) a, \quad n_{3}=n_{1}+n_{2}+1 .
$$

The probability of having such a configuration, $P_{n_{1}, n_{2}}$, depends only on the incoming propagators 1 and 2, which completely fix the configuration. Fur- 
3

\begin{tabular}{|llllllll}
1 & 1 & 1 & 1 & 1 & 1 & 1 & 1 \\
1 & 1 & 1 & 1 & 1 & 1 & 1 & 1 \\
1 & 1 & 1 & 1 & 1 & 1 & 1 & 1 \\
1 & 1 & 1 & 1 & 1 & 1 & 1 & 1 \\
1 & 1 & 1 & 1 & 1 & 1 & 1 & 1 \\
1 & 1 & 1 & 1 & 1 & 1 & 1 & 1 \\
1 & 1 & 1 & 1 & 1 & 1 & 1 & 1 \\
1 & 1 & 1 \\
1 & 1 & 1 \\
1 & 1 & 1 & 1 & 1 & 1 \\
1 & 1 & 1 & 1 & 1 & 1 \\
1 & 1 & 1 & 1 & 1 & 1 \\
1 & 1 & 1 & 1 & 1 & 1 \\
& $n$ & & & 1 & 1 & 1 \\
& & & & & & \\
& & & & &
\end{tabular}

Figure 5: Another Vertex

thermore, in the leading order of the mean field approximation, the probability for the occurence of a collection of solid and dotted lines is the product of the probabilities for the occurence of each individual line. Therefore, we have,

$$
P_{n_{1}, n_{2}}=P_{n_{1}} P_{n_{2}},
$$

where $P_{n}$, the probability for a single propagator is given by

$$
P_{n}=x(1-x)^{n} .
$$

The hypothesis about the factorization of probabilities used to derive the above results implies lack of correlation between different lines. This is in fact the basic hypothesis of the mean field method: To the leading order, each line propagates independently in the background of the mean field $x$, and the correlations between different lines show up only in higher order corrections. 
Putting together eqs. $(45,46,47)$, we have,

$$
V_{+}=\frac{g F(x)}{8}
$$

where

$$
\begin{aligned}
F(x) & =\sum_{n_{1}=0}^{\infty} \sum_{n_{2}=0}^{\infty} \frac{P_{n_{1}, n_{2}}}{\left(n_{1}+1\right)\left(n_{2}+1\right)\left(n_{3}+1\right) a^{3}} \\
& =\sum_{n_{1}=0}^{\infty} \sum_{n_{2}=0}^{\infty} \frac{x^{2}(1-x)^{n_{1}+n_{2}}}{\left(n_{1}+1\right)\left(n_{2}+1\right)\left(n_{1}+n_{2}+2\right) a^{3}} .
\end{aligned}
$$

In Appendix A, it is shown that this sum can be converted into a single integral. After this simplification, we have the following expression for $V_{+}$:

$$
V_{+}=\frac{g x^{2}}{(1-x)^{2} a^{3}} \int_{0}^{1-x}\left(\frac{1}{y}(\ln (1-y))^{2}\right) d y .
$$

At this point, it is possible to write down a fermionic interaction term, using $V_{+}$and $V_{-}=g$. All we have to do is to replace the term $\tilde{g} \bar{\psi} \sigma_{1} \psi$ in eq.(14) by

$$
V_{+} \bar{\psi}_{1} \psi_{2}+V_{-} \bar{\psi}_{2} \psi_{1} .
$$

However, instead of this awkward looking non-symmetric expression, we prefer to use a symmetrized expression. Since in calculating a general graph, $V_{+}$and $V_{-}$come in pairs and always in the form of the product $V_{-} V_{+}$, we are free to redefine individual $V$ 's as we wish, so long as the product remains fixed. A symmetrized expression corresponds to the choice $V_{+}=V_{-}$. This amounts to setting

$$
\tilde{g}^{2}=a V_{+} V_{-} .
$$

The sudden appearence of a factor of $a$ in this equation requires an explanation. Consider a typical solid line (Fig.3), located at some $\sigma=\sigma_{0}$, with factors $V_{+}$and $V_{-}$attached at the ends of the line. As explained earlier, one has to integrate over the position $\sigma_{0}$ of the coordinate. However, in deriving eq. (50) for $V_{+}$, the $\sigma$ coordinate was first latticized with a spacing $a$. Therefore, instead of an integral, we really have a sum over the discretized positions of the solid line. Converting this sum into an integral in the continuum limit introduces a factor of $a$ through

$$
a \sum_{\sigma_{n}} \rightarrow \int d \sigma .
$$


Combining eqs.(44,50,51), we rewrite $\tilde{g}$ as

$$
\tilde{g}=\frac{g x}{a(1-x)}\left(\int_{0}^{1-x}\left(\frac{1}{y}(\ln (1-y))^{2}\right) d y\right)^{1 / 2} .
$$

We note that this expression for $\tilde{g}$ has the correct scaling properties, discussed at the end of section 3. Since $g$ and $x$ are scale invariant constants, $\tilde{g}$ scales as $1 / a$ (see eq.(17)), which is the correct result.

\section{Minimizing The Ground State Energy}

We will now rewrite the ground state energy $E^{-}$(eq.(36)), combining some constants to simplify the expression. We set

$$
\alpha^{2}=1 / a^{\prime}
$$

and define the constant $\gamma$ by

$$
\gamma=2 \pi\left(a / a^{\prime}\right)^{3 / 2}
$$

$\gamma$ stays finite in the limit when both cutoff parameters $a$ and $a^{\prime}$ go to zero, provided that the ratio $a / a^{\prime}$ is kept finite. It is then convenient to eliminate $a^{\prime}$ in favor of $\gamma$ and $a$, and to express $\tilde{g}$ in terms of $x$ through eq.(52), with the result

$$
E^{-}=\frac{D p^{+}}{a^{2}}\left(\gamma \frac{x}{\sqrt{1-x}}-2 g h(x)\right)
$$

where

$$
h(x)=\frac{x^{3 / 2}}{(1-x)^{1 / 2}}\left(\int_{0}^{1-x} \frac{1}{y}(\ln (1-y))^{2} d y\right)^{1 / 2} .
$$

We make a couple of observations regarding this formula: Energy, an extensive quantity, is proportional to $p^{+}$, the length of the $\sigma$ interval, as it should be. It is also proportional to $1 / a^{2}$, and so it diverges in the limit $a \rightarrow 0$. This is not surprising, since $E$ is equal to $p^{-}$, and in the frame $\mathbf{p}=0$ that we have chosen, the product $p^{+} p^{-}$is equal to the square of the mass of the (string) state. If we denote the mass of the lowest string state by $m_{0}$, then

$$
m_{0}^{2}=p^{+} E^{-} .
$$

This is the bare mass, which we expect to be cutoff dependent without renormalization. In the next section, we will see how this cutoff dependence can 


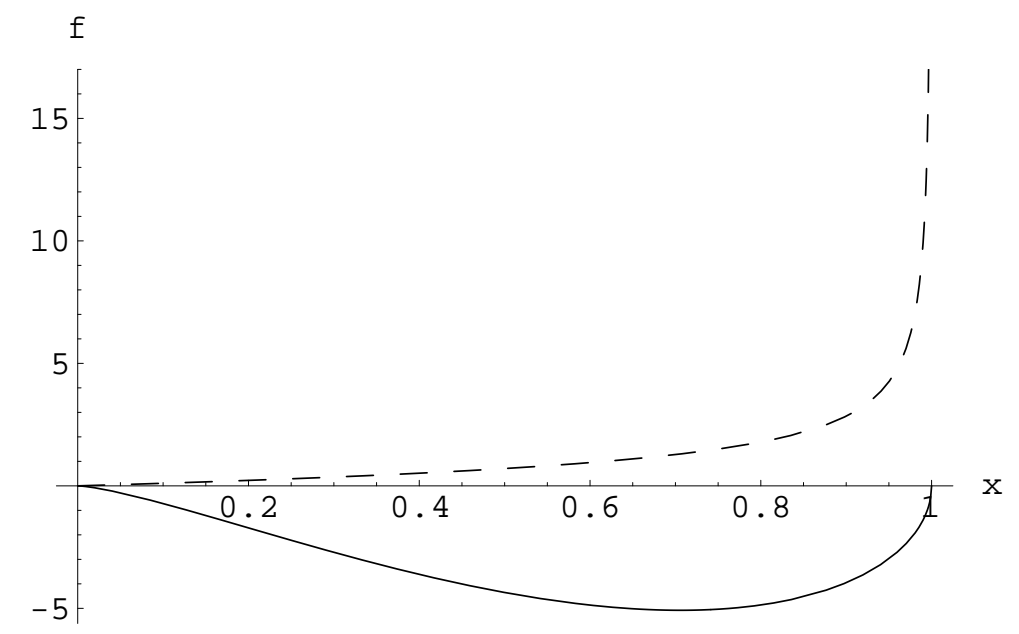

Figure 6: $f(x)=x / \sqrt{1-x}$ is the dashed line and $f(x)=-20 h(x)$ is the solid line

be removed by introducing a suitable mass counter term. Finally, note the difference in the sign of the two terms; this is the key to the existence of a non-trivial minimum. Also, $m_{0}^{2}$, being proportional to $\left(p^{+} / a\right)^{2}$, is scale invariant (see eqs.(6) and (17)). Since mass is a Lorentz invariant quantity, this is as it should be.

Before proceeding further, we note one more simplification: Taking advantage of the freedom to renormalize the coupling constant $g$ and to redefine the cutoff parameter $a$, we can set the constant $\gamma$ equal to unity, so that

$$
E^{-} \rightarrow \frac{D p^{+}}{a^{2}}\left(\frac{x}{\sqrt{1-x}}-2 g h(x)\right) .
$$

However, $\gamma$ is not completely eliminated from the problem. For example, the string slope $\alpha^{\prime 2}$ still depends on $a / a^{\prime}$ and therefore on $\gamma$ (eq.(42)). The bottom line is that, whether one calls it $\gamma$ or $a / a^{\prime}$, one arbitrary constant remains in the problem.

It remains to search for the minimum of $E^{-}$as a function of $x$. In Fig.6, the first term in eq.(56), $x / \sqrt{1-x}$, and the function $-20 h(x)$, the second term for $g=10$, are seperately plotted against $x$. Both curves start at the origin, and for small enough $g$, the first term dominates the second term in absolute value. Therefore, for small $g$, the minimum of $E^{-}$is at $x=0$, $E^{+}=E^{-}=0$, and we have recovered the perturbative field theory as the 


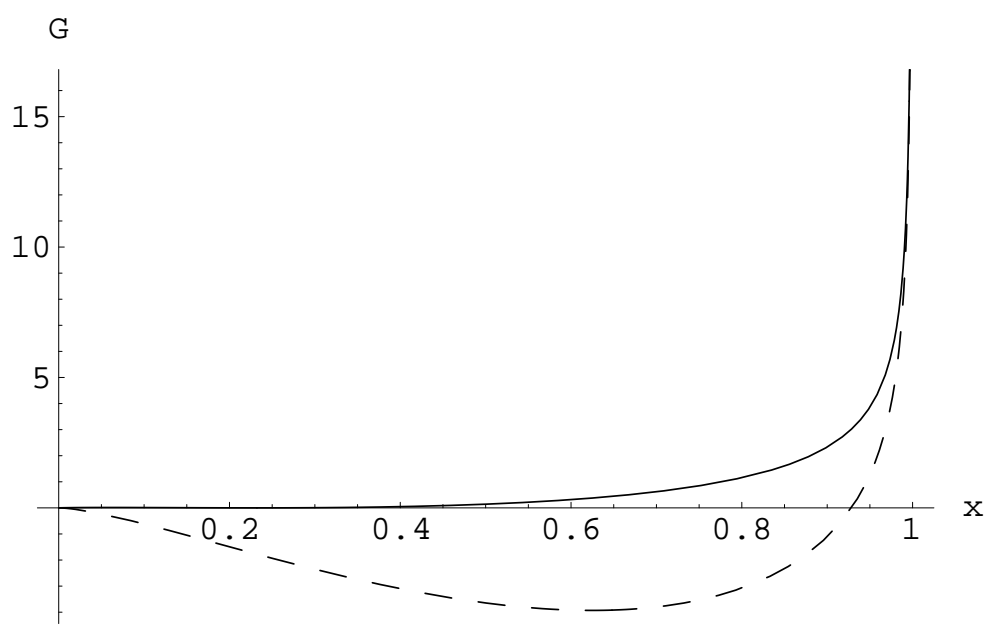

Figure 7: The solid line represents $\mathrm{G}(\mathrm{x})$ at $\mathrm{g}=1.3$ and the dashed line represents $\mathrm{G}(\mathrm{x})$ at $\mathrm{g}=10$

ground state. Stated another way, the only solution to meanfield equations at small coupling constant corresponds to vanishing order parameter $x$, and therefore to the perturbative phase of the underlying field theory. As $g$ gets bigger, there is a turning point around $g \simeq 1.3$, and past this point, the second term dominates. The minimum $E^{-}$now occurs at some $x \neq 0, E^{-}$ is negative at this minimum, and it wins over $E^{+}=0$ as the ground state. Therefore, there is a critical value of $g=g_{c}$, with $g_{c} \simeq 1.3$, such that for $g<g_{c}$, the system is in the perturbative phase, and for $g>g_{c}$, it is in the string phase. In Fig.7, the quantity

$$
G=\frac{a^{2} E_{-}}{D p^{+}}
$$

is plotted against $x$ for $g=1.3$ and also for $g=10$. For the first value of $g \simeq g_{c}$, the minimum is at $x=0$, and for the second one, it is at $x \simeq 0.625$. As $g$ asymptotes to infinity, the location of the minimum asymptotes to $x \simeq 7.06$, which coincides with the location of the minimum of $h(x)$.

To summarize, in this section, we have seen that string formation takes place if the coupling constant is larger than a critical value. However, it is important to realize that so far we have been talking about an unrenormalized theory. The two physical parameters associated with a free string are the slope and the intercept, and these should be finite. We have already seen 


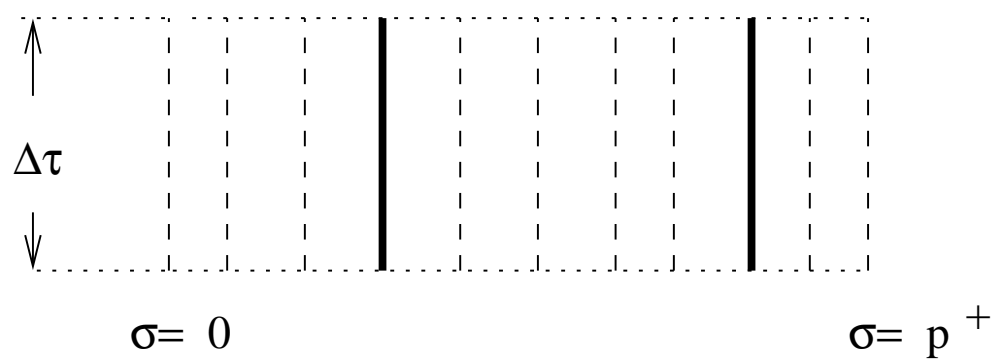

Figure 8: A strip of width $p^{+}$and thickness $\Delta \tau$ on the worldsheet

from eq.(42) that the slope is finite if the ratio of the two cutoff parameters, $a / a^{\prime}$ is finite as $a$ and $a^{\prime}$ tend to zero. On the other hand, the intercept, which is given by $p^{+} E^{-}$(eq.(55)), diverges as $a \rightarrow 0$ because of the factor $1 / a^{2}$ in eq.(56). We will see in the next section that, this divergence can be cancelled by introducing a suitable bare mass (counter)term in the original action.

\section{Non-Zero Mass}

Up to this point, we have taken the mass parameter in the propagator (eq.(2)) to be zero. It is of course important to be able to deal with nonzero mass, since in any case, even if we set the bare mass equal to zero, the renormalized mass will in general be different from zero. In particular, as pointed out in the last section, the mass squared of the lowest string state, the string intercept, is cutoff dependent. We will now show that, by introducing a suitable mass counter term, we can eliminate this cutoff dependence, and tune the intercept to any finite value of our choice. This should be contrasted with what happens in the critical string theory, where the intercept is fixed.

We wish to compute the contribution to the world sheet action of the mass term in the propagator. This calculation is greatly simplified by considering a thin strip of the world sheet (Fig.8), bounded by two lines located at constant $\tau$ and constant $\tau+\Delta \tau$ in the $\tau$ direction, and extending from $\sigma=0$ to $\sigma=p^{+}$in the $\sigma$ direction. Fig. 8 shows a bunch of dotted and solid lines in this strip, representing propagators that propagate for an infinitesimal time interval $\Delta \tau$. The contribution of the mass term to the path integral, for small $\Delta \tau$, is of the form

$$
1+\Delta \tau M
$$


and this can be iterated in the $\tau$ direction to get

$$
\exp \left(\left(\tau_{f}-\tau_{i}\right) M\right),
$$

so it boils down to calculating $M$.

We will do this calculation using the mean field method, along lines similar to the calculation of the vertex in section 6 . Let the number of dotted lines in Fig. 8 be $n$, and the number of solid lines be $N-n$, where $N$, the total number of lines, is fixed by

$$
N=p^{+} / a,
$$

where $p^{+}$is the total width of the strip. Denote the contribution to $M$ for a given value of $n$ by $M_{n}$, and recall that the probability of having a dotted line is $1-x$ and that of having a solid line is $x$. To get $M$, we weigh each configuration $M_{n}$ by the corresponding statistical factor and add:

$$
M=\sum_{n}(1-x)^{n} x^{N-n} M_{n} .
$$

It remains to calculate $M_{n}$, by collecting the mass dependent terms coming from various propagators. The relevant term in eq.(2) can be rewritten as

$$
\exp \left(-\frac{m^{2} \Delta \tau}{2\left(\sigma_{i+1}-\sigma_{i}\right)}\right) \rightarrow 1-\frac{m^{2} \Delta \tau}{2\left(\sigma_{i+1}-\sigma_{i}\right)},
$$

where $\sigma_{i}$ are the $\sigma$ coordinates of the solid lines, with $i=1,2, \ldots, N-n-1$. We note that, in this case, the $p^{+}$in eq.(2) corresponds to $\sigma_{i+1}-\sigma_{i}$, the distance between two adjacent solid lines. Also, we find it convenient to adopt Euclidean metric for this calculation and therefore the factor of $i$ has been dropped. Finally, to get $M_{n}$, one has to sum over the positions of the solid lines

$$
M_{n}=\sum_{\sigma_{i}}\left(-\frac{m^{2}}{2\left(\sigma_{i+1}-\sigma_{i}\right)}\right) .
$$

This sum is evaluated in Appendix B, with the result

$$
M=p^{+} \frac{m^{2} x^{2}}{a^{2}(1-x)} \ln (x) .
$$

Comparing this to the energy with the energy in the absence of bare mass (eq.(56)), we note the common factors of $p^{+}$and $1 / a^{2}$, but we also see that we have to scale the mass by

$$
m^{2} \rightarrow D m^{2}
$$




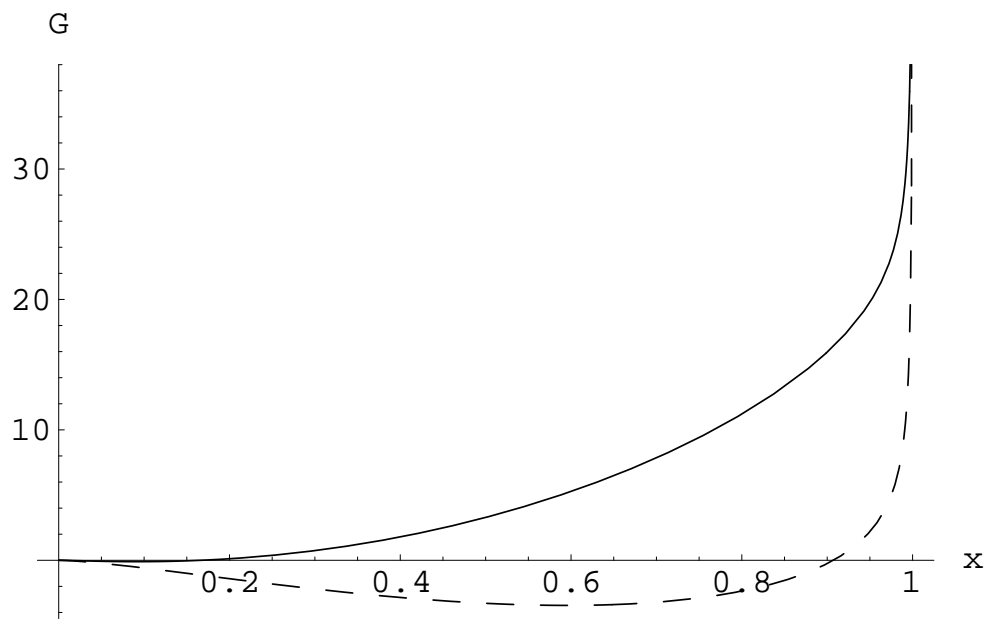

Figure 9: The dashed curve is $\mathrm{G}(\mathrm{x})$ for $m^{2}=1$ and the solid curve is $\mathrm{G}(\mathrm{x})$ for $m^{2}=20$

so that the terms in the expression for the energy all have a common factor of $D$. Otherwise, the mass term would drop out in the large $D$ limit. Combining eqs.(56) and (61), the total energy, including the mass, is

$$
\begin{aligned}
E^{-} & =\frac{D p^{+}}{a^{2}}\left(\frac{x}{(1-x)^{1 / 2}}-2 g \frac{x^{3 / 2}}{(1-x)^{1 / 2}}\left(\int_{0}^{1-x} \frac{\ln ^{2}(1-y)}{y} d y\right)^{1 / 2}\right. \\
& \left.-\frac{m^{2} x^{2}}{1-x} \ln (x)\right) .
\end{aligned}
$$

Since $x \leq 1$, the contribution of the mass term to the total energy is positive. Remembering that the sum of the other two terms was negative, we see that the mass term tends to raise the ground state energy, in agreement with what one would expect.

In Fig.9, $G$ (eq.(57)) is plotted against $x$ for $g=10$, and for two different values of $m^{2}: m^{2}=1$, the dashed curve and $m^{2}=20$, the solid curve. For $m^{2}=1$, the curve is very similar to the one in the massless case: There is non-trivial minimum around $x \simeq 0.6$. At the larger value $m^{2}=20$, the curve flattens and the minimum shifts to $x=0$. This means that string formation takes place only if the mass is not too large, and the coupling constant is large enough. Otherwise, the model is in the perturbative field theory phase.

At this point, it is important to remember that so far, we have been talking only about the cutoff dependent part of the enegy, which is proportional 
to $1 / a^{2}$. Similarly, the bare mass term makes a contribution proportional to $1 / a^{2}$ to the ground state energy. We have seen that string formation takes place if the coupling constant is large enough and the mass is sufficiently small so that ground state energy is negative. We hasten to add that all of this is before renormalization. Renormalization requires that the cutoff dependent part of the ground state energy should be zero. We have seen above that this can be arranged by suitably tuning the bare mass. Now the question is, is there still string formation even after renormalization? We have seen above that zero (cutoff dependent) ground state energy marks the borderline between the string and field theory phases, and so we cannot conclude anything definite on the basis of what we have so far. To decide this question, one has to go beyond the cutoff dependent part of the ground state energy and compute the finite contributions. We have already seen that the second term on right of eq.(25) is one such contribution, but there are also similar finite terms coming from corrections to eq.(52) for $\tilde{g}$ and eq.(61) for M. These calculations are rather involved and they will not be attempted in this article, and therefore, the question of whether there is string formation after renormalization remains open. We hope to return to this problem in the future.

\section{Higher Order Contributions To $S_{1}$}

So far, we have computed the leading term in the action in the large D limit, which is proportional to D. The next order term is D independent, and to compute it, one has to follow the standard prescription of the saddle point method and expand the fields $\kappa_{1,2}$ and $\lambda_{1,2}$ around their mean value, keeping only the quadratic terms. The functional integrals can then in principle be done, producing the desired term in the action. This calculation was carried out in [5]; here, we will briefly review it and also discuss its significance and its renormalization.

We first notice that there are two different sources of higher order terms: Those coming from $S_{1}$ and those coming from the rest of the action, such as the fermionic sector, $\tilde{g}$ and the mass term. The contribution coming from $S_{1}$ has a special significance: It contains the kinetic energy term for a new degree of freedom which was not present in the original action. The rest of the higher order contributions do not seem to have any special significance, so we will not consider them any further.

Consider the effective action resulting from carrying out the functional 
integration over $\mathbf{q}$ in $S_{1}$ (eq. $\left.(23)\right)$ :

$$
S_{1} \rightarrow \frac{i}{2} D \operatorname{Tr} \ln \left(-\partial_{\sigma}^{2}+A^{2} \partial_{\tau}^{2}\right)
$$

where $A^{2}$ can be split into the zeroth order term $A_{0}^{2}$, which is independent of the world sheet coordinates, plus a fluctuating term $\Delta A^{2}$ :

$$
A^{2}=A_{0}^{2}+\Delta A^{2}, \quad A_{0}^{2}=\frac{\kappa_{1,0}^{2}}{\kappa_{2,0}}=\frac{a^{\prime}}{a} \frac{x^{2}}{1-x}, \quad \Delta A^{2}=\frac{a^{\prime}}{a} \frac{2 x-x^{2}}{(1-x)^{2}} \Delta x .
$$

We then expand in powers of $\Delta A^{2}$ in the form of a series

$$
S_{1}=S_{1}^{(0)}+S_{1}^{(1)}+S_{1}^{(2)}+\cdots
$$

Since $\Delta A^{2}$ is expressible in terms $\Delta x$, this expansion can also be converted into an expansion in powers of $\Delta x$.

The leading contribution

$$
S_{1}^{(0)}=\frac{i}{2} D \operatorname{Tr}\left(-\partial_{\sigma}^{2}+A_{0}^{2} \partial_{\tau}^{2}\right)
$$

was already computed in section 4 . Since we are expanding around a saddle point, $S_{1}^{(1)}$ vanishes. The focus of our attention here is the term second order in $\Delta A^{2}$ :

$$
S_{1}^{(2)}=-\frac{i D}{4} \operatorname{Tr}\left(\left(\partial_{\sigma}^{2}-A_{0}^{2} \partial_{\tau}^{2}\right)^{-1} \partial_{\tau}\left(\Delta A^{2}\right) \partial_{\tau}\left(\partial_{\sigma}^{2}-A_{0}^{2} \partial_{\tau}^{2}\right)^{-1} \partial_{\tau}\left(\Delta A^{2}\right) \partial_{\tau}\right)
$$

This term contains both a logarithmically divergent and also a finite part. We will first compute the divergent part, and we will later see that we do not need to know the finite part.

Rewriting it in momentum space, we have

$$
S_{1}^{(2)}=-\frac{i D p^{+}}{16 \pi^{2}} \int d^{2} k^{\prime} I\left(k^{\prime}\right) \Delta \tilde{A}^{2}\left(k^{\prime}\right) \Delta \tilde{A}^{2}\left(-k^{\prime}\right),
$$

where $\Delta \tilde{A}^{2}\left(k^{\prime}\right), k^{\prime}=\left(k_{0}^{\prime}, k_{1}^{\prime}\right)$, is the Fourier transform of $\Delta A^{2}(\tau, \sigma)$, and

$I\left(k^{\prime}\right)=\int d^{2} k \frac{\left(4 k_{0}^{2}-\left(k_{0}^{\prime}\right)^{2}\right)^{2}}{\left(\left(2 k_{1}+k_{1}^{\prime}\right)^{2}-A_{0}^{2}\left(2 k_{0}+k_{0}^{\prime}\right)^{2}\right)\left(\left(2 k_{1}-k_{1}^{\prime}\right)^{2}-A_{0}^{2}\left(2 k_{0}-k_{0}^{\prime}\right)^{2}\right)}$. 
In the the expression for I, we have let $p^{+} \rightarrow \infty$ and replaced the discrete sums over the variables $k_{1}$ and $k_{1}^{\prime}$ by integrals. Clearly, this is permissible when one is calculating an ultraviolet divergent term, which is sensitive only to the large momentum limit.

Next, we expand I in powers of $k^{\prime}$. The zeroth order term was already included in the calculation of $S_{1}^{(0)}$, the first order term vanishes, and terms with powers of $k^{\prime}$ greater than two are convergent. The logarithmic divergence comes exclusively from the quadratic terms, given below:

$$
I \simeq \frac{i \pi}{2 A_{0}^{5}}\left(A_{0}^{2}\left(k_{0}^{\prime}\right)^{2}-\left(k_{1}^{\prime}\right)^{2}\right) \int \frac{d k}{k} .
$$

This integral is both ultraviolet and infrared divergent. The infrared divergence is due to letting $p^{+} \rightarrow \infty$; it can be taken care of by introducing a lower limit of roughly $1 / p^{+}$in the integral over $\mathrm{k}$. To eliminate the ultraviolet divergence, the integral is cutoff at the upper limit $k=\Lambda$, where $\Lambda$ is the same cutoff used in section 4 (eq.(25)), with the result

$$
\int \frac{d k}{k} \rightarrow \ln \left(\Lambda p^{+}\right)
$$

Combining eqs.(66) and (68) and transforming back to the position space gives

$$
S_{1}^{(2)} \simeq \frac{D \ln \left(\Lambda p^{+}\right)}{32 \pi A_{0}^{5}} \int_{0}^{p^{+}} d \sigma \int_{\tau_{i}}^{\tau_{f}} d \tau\left(A_{0}^{2}\left(\partial_{\tau}\left(\Delta A^{2}\right)\right)^{2}-\left(\partial_{\sigma}\left(\Delta A^{2}\right)\right)^{2}\right) .
$$

This equation tells us that $\Delta x$ represents a new propagating degree of freedom, with its own kinetic energy. The promotion of a constrained field into a propagating degree of freedom should be familiar from other two dimensional models [16,17]. Since $x$ is related to the fermionic bilinear $\rho$ through eq.(41), it is reasonable to interpret this new degree of freedom as a bound state of a pair of fermions.

We would like to say a few words about the renormalization of this result. We can get rid of the factor of $D$ and the logarithmic factor if we scale $\Delta A^{2}$ by letting

$$
\Delta A^{2} \rightarrow\left(\frac{16 \pi^{2}}{D \ln \left(\Lambda p^{+}\right)}\right)^{1 / 2} \Delta A^{2}
$$

As a result, this term is now of order zero in the large $D$ expansion, as opposed to terms calculated in the previous sections, which were proportional to $D$. 
Also, the logarithmic divergence has dissappeared. We note that, the finite terms which we have not calculated (see the discussion after eq.(65)), which are also zeroth order in $D$, will all be suppressed by this logarithmic factor. Of course, we still expect contributions from the higher order terms in the large $D$ expansion.

It may be of some interest to express these results by writing down a sigma model. Since the order parameter $x$ has now become a dynamical field, we will rename it $\chi$, and $x$ is now the expectation value of $\chi$. Defining $A(\chi)$ by

$$
A(\chi)=\left(\frac{a^{\prime}}{a}\right)^{1 / 2} \frac{\chi}{(1-\chi)^{1 / 2}},
$$

we combine eq.(70) with eq.(62) to form the sigma model:

$$
S_{\sigma}=\frac{D \ln \left(\Lambda p^{+}\right)}{8 \pi} \int_{0}^{p^{+}} d \sigma \int_{\tau_{i}}^{\tau_{f}} d \tau\left(\frac{\dot{A}^{2}}{A}-\frac{A^{\prime 2}}{A^{3}}-\mathcal{V}(\chi)\right)
$$

To the leading order in $D$, the potential $\mathcal{V}$ in this equation is given by

$$
\mathcal{V}(\chi)=-\frac{1}{p^{+}} E^{-}(\chi)
$$

and $E^{-}$is the ground state energy(eq.(62)), with the argument $x$ replaced by $\chi$.

We note that, the slope parameter is no longer a number, but it is now given by

$$
\alpha^{\prime 2}=\frac{a^{\prime}}{4 a} \frac{\chi^{2}}{1-\chi}
$$

and so it becomes a fluctuating dynamical field. We believe that this is the crucial difference between the fundamental strings of string theory and the field theory strings of the type developed in the present work. In string theory, the slope is fixed, whereas here, it is a dynamical variable. In particular, it can fluctuate and make a transition from the saddle point $x \neq 0$ to the other saddle point $x=0$. We recall from section 5 that this latter saddle point corresponds to perturbative field theory. In the present work, the world sheet configuration we have chosen is a cylinder of infinite extent in the $\tau$ direction. For such a configuration, and for the cutoff theory before renormalization, we have shown that the string forming saddle point at $x \neq 0$ is energetically favored. However, for other configurations of the world sheet, 
the other saddle point at $x=0$ may be more important. For example, the other saddle point may contribute to a world sheet configuration appropriate to a scattering process. What we have in mind is, for example, a high energy and fixed angle scattering process, which is represented by the scattering of the fundamental constituents (partons) of a field theoretic model. It would be very nice if the saddle point $x=0$ was dominated this process, whereas the the other saddle point, $x \neq 0$ dominated the high energy Regge limit. This would then explain how two different mechanisms, one underlying the "hard" high energy scattering and the other underlying the "soft" high energy scattering, could coexist. Inspired by the AdS/CFT correspondence, models of this type have been costructed $[18,19,20]$. It is of interest to note that, in these models also the string (Regge) slope is allowed to fluctuate.

\section{Conclusions}

This article is an extension of the earlier work $[2,3,4,5]$ on summing planar graphs by putting them on the world sheet. Although as in the earlier work, our guinea pig theory is still the $\phi^{3}$ theory and the approximation scheme used is still the mean field method, there is also quite a bit of new material. In the previous work, the prefactor that appears in the world sheet propagator (eq.(2)) had been omitted; here, we rectify that omisssion. Also, up to now, the bare mass of the field $\phi$ was taken to be zero; in this work, we introduce a non-zero bare mass into the problem. Prior to the introduction of the mass, a cutoff was needed to have a well defined model, and some physical quantities, such as the ground state energy, depended on the cutoff. With the introduction of a mass counter term, it becomes for the first time possible to renormalize the model by eliminating the cutoff dependence.

Going back to the cutoff theory, we find string formation for a range of the values of the mass and coupling constant. For the values of these parameters outside this range, the model goes back to the original starting point, namely, perturbative field theory. In the special case of vanishing bare mass, this is in agreement with the resuts of the previous work.

In contrast to the model with cutoff, we know very little about the renormalized model. In the future, we hope to come back to and study it. It would be interesting to find out whether there is string formation for any range of the parameters of the model.

Another interesting problem left open for future research is to deduce the consequences of the promotion of the string slope into a dynamical field. As 
explained at the end of the last section, this could help connect the Regge and parton regimes of high energy scattering processes.

\section{Acknowledgements}

This work was supported in part by the Director, Office of Science, Office of High Energy and Nuclear Physics, of the US Department of Energy under Contract DE-AC03-76SF00098, and in part by the National Science Foundation Grant No.PHY99-07949. Part of the research leading to this article was done while I was attending the program on QCD and Strings at KITP, Santa Barbara. I would like to thank the organizers of this program.

\section{Appendix A}

It is useful to derive an expression for $V_{+}$that does not involve infinite sums. For this purpose, we define an auxilliary function by

$$
\tilde{F}\left(x_{1}, x_{2}\right)=\sum_{n_{1}=0}^{\infty} \sum_{n_{2}=0}^{\infty} \frac{\left(1-x_{1}\right)^{n_{1}}\left(1-x_{2}\right)^{n_{2}}}{\left(n_{1}+1\right)\left(n_{2}+1\right)\left(n_{1}+n_{2}+2\right)} .
$$

The original function can be expressed in terms of $\tilde{F}$ as

$$
F(x)=\frac{x^{2}}{a^{3}} \tilde{F}\left(x_{1}=x, x_{2}=x\right),
$$

so the problem reduces to evaluating $\tilde{F}$.

It is easy to show that $\tilde{F}$ satisfies the following differential equation:

$$
\begin{aligned}
& \left(2-\left(1-x_{1}\right) \frac{\partial}{\partial x_{1}}-\left(1-x_{2}\right) \frac{\partial}{\partial x_{2}}\right)\left(1-\left(1-x_{1}\right) \frac{\partial}{\partial x_{1}}\right)\left(1-\left(1-x_{2}\right) \frac{\partial}{\partial x_{2}}\right) \tilde{F}\left(x_{1}, x_{2}\right) \\
& =\frac{1}{x_{1} x_{2}}
\end{aligned}
$$

This differential equation can be integrated partially to give

$$
\left(2-\left(1-x_{1}\right) \frac{\partial}{\partial x_{1}}-\left(1-x_{2}\right) \frac{\partial}{\partial x_{2}}\right) \tilde{F}\left(x_{1}, x_{2}\right)=\frac{\ln \left(x_{1}\right) \ln \left(x_{2}\right)}{\left(1-x_{1}\right)\left(1-x_{2}\right)},
$$

and a further integration leads to the result

$\tilde{F}=\frac{1}{\left(1-x_{1}\right)\left(1-x_{2}\right)} \int_{0}^{2-x_{1}-x_{2}} \frac{d y}{y} \ln \left(1-\frac{y\left(1-x_{1}\right)}{2-x_{1}-x_{2}}\right) \ln \left(1-\frac{y\left(1-x_{2}\right)}{2-x_{1}-x_{2}}\right)$. 


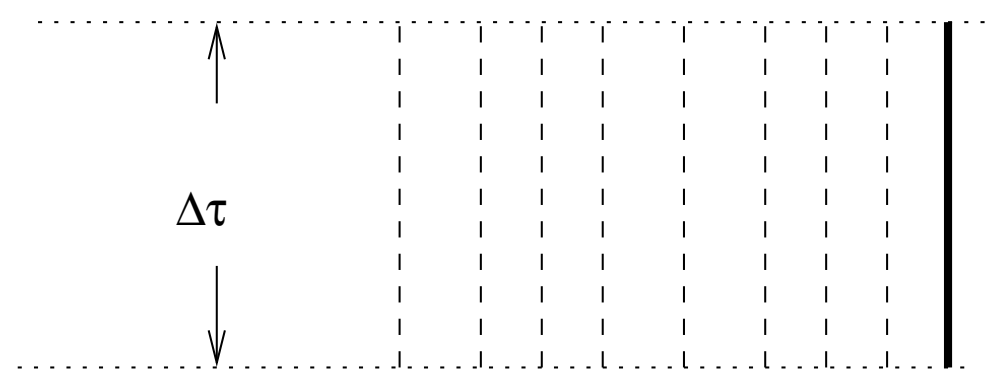

Figure 10: A special configuration of lines

Finally, substituting this result for $\tilde{F}$ in eqs.(74) and (48), we get the following expression for $V_{+}$:

$$
V_{+}=\frac{g x^{2}}{(1-x)^{2} a^{3}} \int_{0}^{1-x} \frac{d y}{y}(\ln (1-y))^{2} .
$$

\section{Appendix B}

In this Appendix, we will present a derivation of eq.(61), starting with eqs.(58) and (60). Instead of trying to do the statistical sum of eq.(58) for a general configuration of solid and dotted lines, we will first consider the simple configuration, shown in Fig.10, of one solid line at the beginning, and all the rest dotted lines. We will show that the general configuration can be reached by iterating this special configuration. Also recall from section 8 that we have a thin strip of width $\Delta \tau$ in the $\tau$ direction. The mass contribution coming from all configurations of this type to the path integral is given by

$$
\tilde{Z}(x)=\sum_{n=1}^{\infty} P_{n}(x)\left(1-\frac{m^{2} \Delta \tau}{n a}\right),
$$

where $n$ is the total number of lines and we have expanded to first order in $\Delta \tau$. The mean field ansatz, eq.(47), gives

$$
P_{n}(x)=x(1-x)^{n-1}
$$

and substituting in (78), we have

$$
\tilde{Z}(x)=1+\frac{m^{2} x \Delta \tau}{a(1-x)} \ln (x) .
$$


So far, we have been summing over an arbitrary number of lines. However, it will prove convenient to select a fixed total number $n$ of lines from the sum. This easily accomplished by introducing a factor of $w$ that keeps track of the number of lines, and letting

$$
K_{n}(x) \rightarrow K_{n}(w, x)=x w^{n}(1-x)^{n-1} .
$$

Eq.(80) is now replaced by

$$
\tilde{Z}(w, x)=\tilde{Z}_{0}(w, x)+\Delta \tau \tilde{Z}_{1}(w, x),
$$

where,

$$
\begin{aligned}
& \tilde{Z}_{0}=\frac{w x}{1-w(1-x)} \\
& \tilde{Z}_{1}=\frac{m^{2}}{a} \frac{x \ln (1-w(1-x))}{1-x} .
\end{aligned}
$$

To isolate the contribution coming from a configuration with $n$ lines, one has to expand in powers of $\mathrm{w}$ and pick the coefficient of $w^{n}$.

Now consider a general configuration of lines, such as in Fig.8. Such a general configuration can be built from the special configuration discussed above (Fig.10) as follows: First iterate the special configuration as a geometric series

$$
\sum_{n=0}^{\infty}(\tilde{Z}(w, x))^{n}=\frac{1}{1-\tilde{Z}(w, x)},
$$

and then add to this a sum over arbitray number of dotted lines given by

$$
\sum_{n=0}^{\infty} w^{n}(1-x)^{n}=\frac{1}{1-w(1-x)} .
$$

The result is then the contribution of the general configuration to the path integral:

$$
Z(w, x)=\frac{1}{(1-\tilde{Z}(w, x))(1-w(1-x))} .
$$

We can now extract $M$ (eq.(58)) from this result as follows: First, pick the term linear in $\Delta \tau$. And then fix the total number of lines to be

$$
N=p^{+} / a,
$$


by expanding in $\mathrm{w}$ and picking the coefficient of $w^{N}$, with the result

$$
\begin{aligned}
M & =\left(\sum_{n=1}^{\infty}(n-1)\left(\tilde{Z}_{0}\right)^{n-1} \frac{\tilde{Z}_{1}}{1-w(1-x)}\right)_{w^{N}}=\left(\frac{\tilde{Z}_{0} \tilde{Z}_{1}}{\left(1-\tilde{Z}_{0}\right)^{2}(1-w(1-x))}\right)_{w^{N}} \\
& =\frac{m^{2} x^{2}}{a(1-x)}\left(\frac{w \ln (1-w(1-x))}{(1-w)^{2}}\right)_{w^{N}} .
\end{aligned}
$$

Expanding in powers of $\mathrm{w}$ gives

$$
\left(\frac{w \ln (1-w(1-x))}{(1-w)^{2}}\right)_{w^{N}}=\sum_{n=1}^{N-1}\left(1-\frac{N}{n}\right)(1-x)^{n} .
$$

This result can be simplified by noticing that as $a \rightarrow 0, N \rightarrow \infty$. Therefore, the first term in parenthesis on the right hand side is negligible compared to the second term, which is proportional to N. Also, the upper limit of the sum can be changed from $N-1$ to $\infty$. Therefore, as $N \rightarrow \infty$,

$$
\left(\frac{w \ln (1-w(1-x))}{(1-w)^{2}}\right)_{w^{N}} \rightarrow-N \sum_{n=1}^{\infty} \frac{1}{n}(1-x)^{n}=N \ln (x) .
$$

Substituting this, with $N=p^{+} / a$, in eq.(85) for $\mathrm{M}$ gives eq.(61), which was to be derived.

\section{References}

1. K.Bardakci and C.B.Thorn, Nucl.Phys. B 626 (2002) 287, hep-th/0110301.

2. K.Bardakci and C.B.Thorn, Nucl.Phys. B 652 (2003) 196, hep-th/0206205.

3. K.Bardakci and C.B.Thorn, Nucl.Phys. B 661 (2003) 235, hep-th/0212254.

4. K.Bardakci, Nucl.Phys. B 667 (2004) 354, hep-th/0308197.

5. K.Bardakci, Nucl.Phys. B 698 (2004) 202, hep-th/0404076.

6. C.B.Thorn, Nucl.Phys. B 637 (2002) 272, hep-th/0203167.

7. S.Gudmundsson, C.B.Thorn and T.A.Tran, Nucl.Phys. B 649 (2003) 3, hep-th/0209102.

8. C.B.Thorn and T.A.Tran, Nucl.Phys. B 677 (2004) 289, hep-th/0307203. 
9. G.'tHooft, Nucl.Phys. B 72 (1974) 461.

10. J.M.Maldacena, Adv.Theor.Math.Phys. 2 (1998) 281, hep-th/9711200

11. O.Aharony, S.S.Gubser, J.Maldacena, H.Ooguri and Y.Oz, Phys.Rep. 323 (2000) 183, hep-th/9905111.

12. C.B.Thorn, Nucl.Phys. B 699 (2004) 427, hep-th/0405018.

13. For a recent review of the large $\mathrm{N}$ method, see M.Moshe and J.ZinnJustin, Phys.Rept. 385 (2003) 69, hep-th/0306133.

14. B.Sakita and M.A.Virasoro, Phys.Rev.Lett. 24 (1970) 1146.

15. D.B.Fairlie and H.B.Nielsen, Nucl.Phys. B 20 (1970) 637.

16. D.Gross and A.Neveu, Phys.Rev. D 10 (1974) 3235.

17. A.D'Adda, M.Luscher and P.Di Vecchia, Nucl.Phys. B 146 (1978) 63.

18. J.Polchinski and M.J.Strassler, hep-th/0003136, JHEP 0305, (2003) 012, hep-th/0209211, Phys.Rev.Lett. 88 (2002) 031601, hep-th/0109174.

19. R.C.Brower and C.I.Tan, Nucl.Phys. B 662 (2003) 393, hep-th/0207144.

20. O.Andreev and W.Siegel, hep-th/0410131. 